\title{
The British Columbia Drug Overdose and Alert Partnership: Interpreting and sharing timely illicit drug information to reduce harms
}

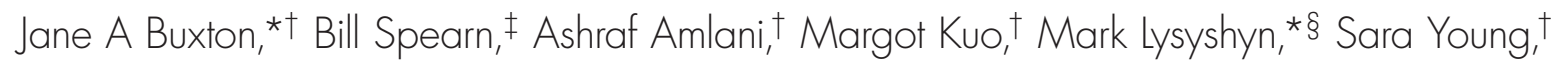 \\ Roy Purssell, \# Kristi Papamihali, ${ }^{\dagger}$ Christopher Mill, ${ }^{\dagger l l}$ and Aaron Shapiro, ${ }^{*}$, on behalf of the B.C. \\ Drug Overdose and Alert Partnership
}

This article is related directly to the Law Enforcement \& Public Health (LEPH) Conference in Toronto, Canada, October 2018.

\begin{abstract}
Illicit drug overdose is a public health issue that leads to significant morbidity and mortality. In order to reduce the harm associated with substance use, emergent issues related to substances and substance use must be addressed in a timely manner, which requires inter-sectoral collaboration. We describe the British Columbia Drug Overdose and Alert Partnership, an innovative collaborative model of stakeholders who work in prevention, harm reduction, treatment and enforcement related to psychoactive substance use. We describe the formation, purpose, stakeholders, and operation of the partnership and resultant public health surveillance system. We use the example of fentanyl-associated overdoses and deaths to describe the attributes that make the system effective. These include timeliness, flexibility, acceptability and costs. This model of inter-sectoral collaboration and surveillance can be applied to other organizations involved in assessing and responding to drug-related harms.
\end{abstract}

Key Words Harm reduction; inter-sectoral collaboration; substance use.

Journal of CSWB. 2019 April;4(1):4-9

www.journalcswb.ca

\section{BACKGROUND}

Overdose due to psychoactive substance use is a public health issue that results in significant morbidity and mortality. Death and other severe health outcomes can be prevented by comprehensive harm-reduction and treatment strategies, such as provision of harm-reduction supplies, education about safer drug use techniques, take-home naloxone programs (Irvine et al., 2018), supervised consumption and overdose prevention sites (Marshall et al., 2011; Wallace, Pagan, \& Pauly, 2019) and oral and injectable opioid agonist treatment (Eibl et al., 2017). While these efforts play important roles in addressing the known harms associated with illicit substances, the unregulated nature of the illicit drug market produces emerging risks that require urgent responses to prevent further harms.

The aim of this paper is to describe the formation and purpose of the Drug Overdose and Alert Partnership (DOAP), a multi-sectoral partnership that collaboratively monitors emerging risks in the illicit drug supply in British Columbia, Canada. We review the member organizations (stakeholders), data sources, and operations of the partnership. We then provide a case example of fentanyl-associated overdoses to illustrate the ability of DOAP to detect and respond to "an outbreak" and then describe some of the attributes that make the system effective, including timeliness, flexibility, acceptability, and cost (Buehler et al., 2004).

\section{Formation and Purpose of the Drug Overdose and Alert Partnership}

Prior to 2011, the Vancouver chapter of the Canadian Community Epidemiology Network on Drug Use had developed informal partnerships to monitor local trends of illicit drug use (Canadian Centre on Substance Use and Addiction, 2019). Previous responses to drug-related harms in British Columbia involved the collaboration of public health, enforcement, and other health partners. For example, two outbreaks of leukoencephalopathy linked to heroin inhalation were investigated in British Columbia between 2001 and 2006; although the etiologic agent was not identified, the distribution of cases in time and place and the identification of two case-couples 
suggested the risk factors were substance-related rather than due to genetic predisposition (Buxton et al., 2012). In 2008, public health officials in western Canada issued public health advisories when severe neutropenia was determined to be associated with levamisole-tainted cocaine (Knowles et al., 2009). A case-control study with genotyping confirmed that the severe neutropenia was genetically determined (Buxton et al., 2015).

In May 2011, the B.C. Coroners Service identified an increase in illicit overdose deaths due to an increase in the purity of heroin. The British Columbia Centre for Disease Control (BCCDC) led an effort to meet with representatives from various sectors (including health, emergency health services, and enforcement) to exchange knowledge and discuss how the issue could have been identified sooner. Concerns were also expressed about the Coroners Service's public safety warning that "higher potency heroin" was circulating in the province. Assumptions were made by people who use drugs (PWUD) and other stakeholders that the heroin was in Vancouver, when it was in fact circulating in another region. Concerns were expressed that the warning may encourage people to seek the potent heroin. This highlighted the need for an effective, ongoing, province-wide partnership for routine surveillance activities and alerting in order to ensure delivery of communications with timely, accurate, and appropriate messaging (Soukup-Baljak et al., 2015).

Responding to emerging risks in the drug supply requires inter-sectoral collaboration between public health officials, toxicology laboratories, law enforcement officials, health-care workers, and people who use drugs, who are often the first to identify an issue and provide insights on how and with whom to communicate. Thus, the British Columbia DOAP was formed in 2011. The goal of DOAP developed by members was "To coordinate stakeholder communication and actions to enable timely alerting and responses to illicit drug use issues."

\section{Membership, Data Sources, and Operation}

The B.C. DOAP is an inter-sectoral multi-level collaborative partnership of stakeholders who work in prevention, treatment, harm reduction, and enforcement related to psychoactive substance use at the local, regional, provincial, and federal levels. The partnership's members are shown in Figure 1; members routinely share information and emerging concerns from their agency/organization, which contributes to a provincial surveillance and alerting system. "Public health surveillance is the ongoing, systematic collection, analysis, interpretation and dissemination of data about a health-related event for use in public health action to reduce morbidity and mortality and to improve health" (German, 2001). The surveillance system developed through DOAP can provide an early warning for, and inform timely response to, emerging issues related to psychoactive substance use.

The source, description, and frequency of posting of ongoing, systematic drug overdose-related data are described in Table 1. Data is shared with DOAP members and their organizations on a password-protected website. Members are informed by e-mail when new data or alerts are posted. The website also serves as a communication forum between members. It has evolved over time to support the needs of the partnership and now includes a message board to post

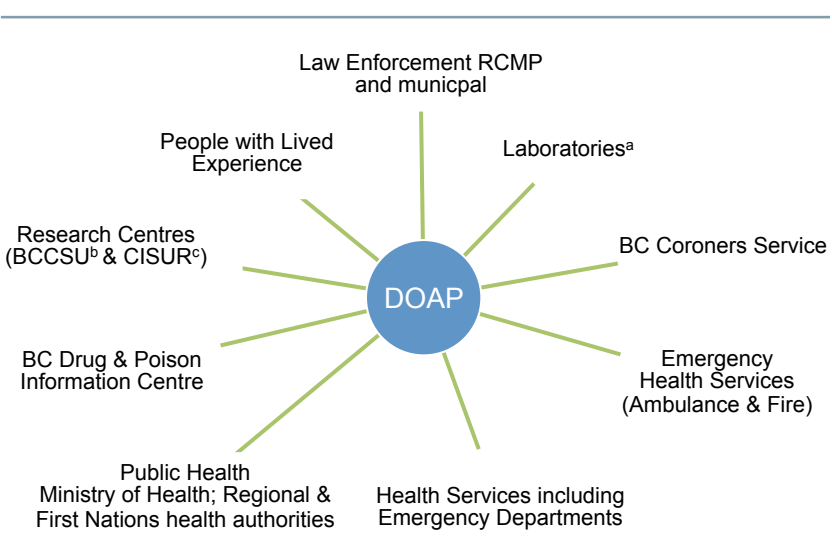

FIGURE 1 Drug Overdose and Alert Partnership (DOAP) member agencies. a Testing Laboratories include Health Canada Drug Analysis Service - enforcement samples (for prosecution or urgent samples as requested by health); Provincial Toxicology Laboratory - decedent samples for BC Coroners Service and drug checking; Hospital and private laboratories patient samples, e.g., urinalysis of people on opioid agonist treatment for health services. DOAP = Drug Overdose and Alert Partnership; BCCSU = B.C. Centre for Substance Use; includes cohort studies and drug checking; CISUR = Canadian Institute for Substance Use Research.

immediate concerns and questions for other DOAP members, a section with alerts and news, and emergency contacts and protocols for responses to unusual events.

Regular meetings serve as a platform for stakeholders to share, review, and interpret information from different geographic regions of British Columbia and from different perspectives. Meetings initially were held quarterly, but in the light of the overdose crisis, members requested more frequent meetings, which now occur every eight weeks. Additional ad hoc communication and meetings occur as needed. In addition to surveillance data being reviewed, research findings and reports from the community are presented and discussed.

While all organizations are committed to the partnership, the level of engagement of individual members may vary based on the immediate priorities, interests, and needs of the member organizations and the populations they serve. However, meeting attendance reflects the members' commitment and the perceived usefulness of the partnership, with more than 30 members attending the past three meetings.

\section{DOAP AS A SURVEILLANCE SYSTEM}

\section{Case Example: Fentanyl-Associated Overdoses}

Fentanyl is a synthetic opioid that is used to relieve severe pain. It is prescribed for pain management in the community only as a transdermal sustained release patch (Young, 2015). Fentanyl is 50 to 100 times more potent than morphine, and some fentanyl analogues may be even more potent, greatly increasing the risk of accidental overdose (Higashikawa \& Suzuki, 2008). Life-threatening respiratory depression occurs more rapidly with fentanyl than with other opioids (Green \& Gilbert, 2016).

In 2012, the B.C. Coroners Service reported fentanyl was detected in $4 \%$ of total unintentional illicit overdose deaths $(n=12)$; this increased to $15 \%(n=50)$ in 2013 (Ministry of 
Source and description of data shared by the B.C. Drug Overdose and Alert Partnership members

\begin{tabular}{|c|c|c|}
\hline Data Source & Data Description & Frequency \\
\hline B.C. Emergency Health Services & $\begin{array}{l}911 \text { calls coded by dispatch as ingestion poisoning by week and location. } \\
\text { An algorithm identifies alerts }{ }^{a}\end{array}$ & Weekly \\
\hline \multirow[t]{2}{*}{ B.C. Centre for Disease Control } & $\begin{array}{l}\text { Dashboard: } \\
\text { i) deaths by health region } \\
\text { ii) Illegal drug overdoses attended by B.C. Emergency Health Services by health } \\
\text { region and city and percentage transported to hospital; } \\
\text { iii) dispatch data is used to derive severity and to project illegal drug overdoses }\end{array}$ & Weekly \\
\hline & $\begin{array}{l}\text { Take Home Naloxone and Facility Overdose Response Box program distribution and } \\
\text { administrations }\end{array}$ & Monthly \\
\hline B.C. Centre for Substance Use & Drug checking reports & Monthly \\
\hline \multirow[t]{2}{*}{ BC Coroners Service } & $\begin{array}{l}\text { Provisional Illicit Drug Overdose Deaths by health region and city, age group, sex, } \\
\text { place of injury, and fentanyl detected }\end{array}$ & $\begin{array}{l}\text { Monthly received } \\
2-3 \text { days prior } \\
\text { to public release }\end{array}$ \\
\hline & $\begin{array}{l}\text { Other reports, e.g., drug type summary reports, mapping by local health area and } \\
\text { detailed reports }\end{array}$ & $\begin{array}{l}\text { As available } \\
\text { or requested }\end{array}$ \\
\hline $\begin{array}{l}\text { B.C. Drug and Poison Information } \\
\text { Centre (DPIC) }\end{array}$ & $\begin{array}{l}\text { Calls to DPIC for exposures to six substance classes (alcohol, benzodiazepines, hal- } \\
\text { lucinogens, opioids, sedative hypnotics, and stimulants) by health region }\end{array}$ & Weekly \\
\hline \multirow{3}{*}{$\begin{array}{l}\text { Health Canada Drug Analysis } \\
\text { Service }\end{array}$} & Line list of drug seizure sample testing results by province & Monthly \\
\hline & Drug situation summary report for Canada and by province & Quarterly \\
\hline & $\begin{array}{l}\text { Alert when new substance found in a region for the first time, with date substance } \\
\text { received }\end{array}$ & As occurs \\
\hline \multirow[t]{2}{*}{ Vancouver Coastal Health } & $\begin{array}{l}\text { Overdose visits by substance type (heroin, other known drug, medication, unknown, } \\
\text { alcohol) to Vancouver Coastal Health emergency departments }(9 / 13 \text { acute care } \\
\text { facilities) }\end{array}$ & Weekly \\
\hline & $\begin{array}{l}\text { Overdoses at Vancouver's supervised consumption site by reported substance used } \\
\text { (heroin, other unknown drug) and intervention (+/-naloxone) and visits and overdoses } \\
\text { at overdose prevention services in Vancouver Coastal Health }\end{array}$ & \\
\hline
\end{tabular}

a ngestion poisoning = any toxic substance including prescribed and over the counter medication, street drugs, and chemicals. Public Health Intelligence for Disease Outbreaks (PHIDO) alerts are reported as low, medium, and high by B.C. geographic areas: local health area (45), health service delivery area (16), and health authority (5) for the last 7, 14, 21, and 28 days.

Public Safety and Solicitor General, 2018). The Royal Canadian Mounted Police (RCMP) and municipal police forces in British Columbia identified fentanyl, both as a white powder mixed with heroin and also in tablet form, as counterfeit oxycodone (fake oxy) tablets containing variable amounts of fentanyl (Canadian Centre on Substance Abuse, 2013; Canadian Centre on Substance Abuse, 2014). Individuals using these tablets would not be aware that they contained fentanyl and were therefore at high risk of accidental overdose. In June 2014, the B.C. Coroners Service warned of increased deaths related to illicit fentanyl use in the Fraser region over the first four months of the year (BC Coroners Service, 2014).

A sudden increase in overdoses at Insite, a supervised consumption site in Vancouver, during a long weekend in October 2014 resulted in a notification to the Vancouver Police Department, who then issued a media release (Vancouver Police Department, 2014a). The police were in contact with local public health officials, who immediately informed the B.C. Emergency Health Service and local emergency departments. Posters informing people who use drugs of the issue were distributed in the community. Those who overdosed reported using "heroin"; however, Health Canada Drug Analysis Service laboratory testing identified only fentanyl and caffeine in samples of the potentially implicated drug obtained by police. Police then released an update stating, "Drug samples taken after a recent rash of suspected heroin overdoses have come back from Health Canada labs as straight Fentanyl" (Vancouver Police Department, 2014b). Following the increase in overdoses, the demand for overdose prevention, recognition, and response training and takehome naloxone (THN) kit distribution doubled compared with the previous month (Toward the Heart, 2019).

All agencies and partners involved in the emergency response to fentanyl-associated overdoses were active DOAP members and were aware of the increasing contamination of drugs with fentanyl through information sharing facilitated by DOAP meetings and resources. Participation in DOAP also enabled comfort and familiarity between members, ensuring commitment to information sharing during such events. This example illustrates how a public health surveillance system 
that combines quality population-level data and strong community collaborations enables a flexible, timely response to reduce harm and improve health outcomes.

\section{Evaluation of DOAP as a Surveillance System}

We use the U.S. Centers for Disease Control framework for evaluating public health surveillance systems for early detection of outbreaks to describe the DOAP surveillance system and the key attributes that contribute to its effectiveness (Buehler et al., 2004).

1. Timeliness is measured by the lapse of time from exposure to the disease agent to the initiation of a public health intervention. Within hours of the overdose events, through established DOAP member communication networks and protocols, supervised consumption site staff notified the Vancouver Police Department, who informed local public health officials and issued a media release. Public health officials in turn communicated with emergency services, and staff and user-groups in the area collaboratively developed warning posters and posted them.

2. Flexibility of a surveillance system refers to the system's ability to adapt to needs. The B.C. DOAP has been effective in communicating different types of substancerelated warnings. Alerts include those regarding content of particular substances such as counterfeit Xanax containing fentanyl, lean, or drank (a red or purple liquid which was confirmed to contain cyclopropylfentanyl), and a cluster of overdoses related to fentanyl in crack cocaine (Klar et al., 2016).

3. Acceptability is reflected in the willingness of participants and stakeholders, including health authorities, the B.C. Coroners Service, the Drug and Poison Information Centre (DPIC), Health Canada Drug Analysis Service laboratory, and emergency health services, to contribute to the data collection and analysis as shown in Table 1. Partners have shared urgent and emergent issues through the website and report regularly consulting the website as a place to access the most recent data.

4. Costs. DOAP is not funded independently; rather, each member organization involved contributes by designating a member to sit on the committee and regularly share data collated by the organization. The BCCDC, within its mandated provincial surveillance activities, chairs and hosts the meetings, provides administrative support, manages the website, and is the central point of contact for members.

\section{Other DOAP Activities}

\section{Shared Data}

To ensure messages and numbers shared publicly are consistent between partners, the latest data is available on the website; a PowerPoint presentation on the website includes slides with the latest numbers and trends for members to use publicly and is updated every two months prior to the DOAP meeting.

\section{Public Safety Campaign}

The proportion of fentanyl-detected deaths in 2014 increased to $25 \%$, from $4 \%$ in 2012. Review of B.C. Coroner Service data identified deaths occurring in young adults who did not inject drugs. This led to an emergency teleconference in January 2015 with DOAP members. Here, the B.C. Coroners Service shared key insights from case investigations, and municipal and federal law enforcement officials shared intelligence regarding the sources of illicitly produced fentanyl. A key decision was made for stakeholders to work together towards developing a targeted public safety campaign using social marketing tools and resources. A working group was struck to identify the target audience, develop and test messages, and plan marketing.

Posters were printed and distributed to partners to post in public settings. A website was developed to provide factual information on fentanyl, including tips on prevention, harm reduction and treatment. DOAP members launched the Know Your Source? Be Drug Smart campaign with a press conference that captured the interest of all major B.C. print, $\mathrm{TV}$, and radio news outlets (Know your source, 2019). The intent of the campaign was to encourage people to reflect whether they really knew where their drugs came from. The campaign has now been replicated in other provinces and territories.

\section{DOAP Opioid Overdose Response Strategy}

On February 4, 2016, the partnership released the DOAP Opioid Overdose Response Strategy (BCCDC, 2016a). The strategy provided recommendations for action that included increased access to naloxone through changes in practice and policy, improving overdose prevention education, training, and services, and enhancing surveillance and utilization of overdose data. On April 14, 2016, the B.C. Provincial Health Officer declared a Public Health Emergency due to opioid overdoses, which facilitated information sharing and enabled further interventions to be implemented (BC Gov News, 2016).

DOAP developed response protocols for unusual drugrelated events, for reporting pharmacy break-ins, and for communicating drug alerts to service providers and the public (BCCDC, 2016b). These protocols have been developed over time in response to identified issues, with expertise and input from stakeholders, including people who use drugs, and building on the group's experiences from each situation. As drug-related issues emerge, DOAP members identify new avenues of surveillance and research to address gaps and improve the evidence base for making informed decisions. New substances identified are discussed with DPIC and toxicologists, and a subgroup reviews toxicology data from various sources to determine co-occurrence of substances and determine whether further action is needed. Collaborations also include emergency medicine physicians who have developed an independent working group.

\section{DISCUSSION}

The time between identification of an illicit substance-related issue and sharing with partners has been reduced dramatically since DOAP was created, such that communication between partners often occurs the same day the event is identified, allowing for more timely responses.

Any inter-sectoral collaboration requires certain conditions to successfully improve health outcomes. These conditions include a shared vision, strong relationships among partners, an effective mix of partners, leadership, adequate resources, efficient structures, and responsive processes 
(Danaher, 2011). We believe DOAP has evolved over the years to harness these enabling conditions. For instance, each DOAP member agency has a different individual mandate around substance use, but the core unifying principle is keeping people, families, and communities safe from drug harms. This powerful shared vision, combined with the inclusiveness and transformational leadership style used in facilitating DOAP, attracts a diverse group of stakeholders, from peers to law enforcement. The ground rules of respectful engagement and strong facilitation skills are needed to balance the power dynamics between partners and foster meaningful discussions.

The expansion and evolution of DOAP has faced challenges. As roles and representatives from participating agencies change regularly, it is necessary to maintain engagement by meeting and reorienting new members. Additionally, as the partnership expanded, peers became less well-integrated in the process. To ensure meaningful participation of peers, a peer consultation group was created. The group meets weekly and can be solicited for input regarding issues that emerge from DOAP as well as providing their own concerns to be raised at DOAP meetings (Greer et al., 2016). Engaging peers in the decision-making process and overdose response ensures proposed interventions and harm-reduction services are relevant and acceptable.

Although law enforcement and public health agencies both have public safety mandates, their approaches to protecting the public from harms associated with illicit drugs may differ. The former focuses on enforcing drug laws and supply reduction, while the latter attempts to engage with marginalized populations through a variety of harm-reduction and addiction-treatment services and peer-based education. Thus, one might expect a collaboration that includes law enforcement officials, public health, health-care workers, and people who use drugs to be challenging. However, given the sustained interest, active participation from members, and expansion of surveillance efforts since DOAP was developed, we conclude that DOAP is highly acceptable and fills a unique niche for monitoring and responding to emerging issues related to psychoactive substance use.

Reports from the front line serve as motivators for members who are removed from the reality on the ground, while presentation of new research, often before publication, helps those engaged in the front line to contextualize their experiences and keep up to date with the latest evidence. Using tools like the DOAP webpage and having administrative support through the BCCDC allows partner agencies to stay informed and engaged without large amounts of e-mail communication or delays seeking permission to share data. Posting of the latest data and presentations with the emerging information in one place ensures the partners share the most recent and consistent data. Finally, having people with lived experience at the table helps identify the most important issues to the community, helps dispel myths, and promotes a learning environment, all of which encourages collaborators to be open-minded and challenges their assumptions.

\section{CONCLUSION}

DOAP provides a forum for multi-level collaboration between actors in the health and law enforcement sectors, ensuring timely communication and interventions for emerging risks arising from illicit drugs. The partnership works collaboratively to monitor trends in substances and substance use and to respond to life-threatening concerns, as illustrated in the case example of fentanyl-associated overdoses. This model of inter-sectoral collaboration and surveillance can be applied to other organizations involved in assessing and responding to drug-related harms. Future evaluations should include qualitative interviews and focus groups with partners, which may help to improve the effectiveness of the DOAP collaboration.

CONFLICT OF INTEREST DISCLOSURES

The author declares there are no conflicts of interest.

\section{AUTHOR AFFILIATIONS}

*Faculty of Medicine, University of British Columbia, Vancouver, BC; ${ }^{\dagger}$ British Columbia Centre for Disease Control, Vancouver, BC; tVancouver Police Department, Vancouver, BC; §Vancouver Coastal Health, Vancouver, BC; \#British Columbia Drug and Poison Information Centre, Vancouver, BC; "Public Health Agency of Canada, Ottawa, ON; IIProvincial Toxicology Centre, Vancouver, BC.

\section{REFERENCES}

BC Centre for Disease Control. (2016a). BC DOAP Opioid Overdose Response Strategy (DOORS). Retrieved from: http://www.bccdc. $\mathrm{ca} /$ resource-gallery/Documents/Opioid\%200verdose\%20 Response\%20Strategy.pdf

BC Centre for Disease Control. (2016b). BCCDC Communicating Drug Alert Guidelines. Retrieved from: http://www.bccdc.ca/resourcegallery/Documents/Educational\%20Materials/Epid/Other/CDA Guidleines_FINALwolink.pdf

BC Coroners Service. (2014, June 4). Coroners Service warns of deaths related to illicit fentanyl use. Retrieved from: https://www2.gov. bc.ca/assets/gov/birth-adoption-death-marriage-and-divorce/ deaths/coroners-service/news/public-safety-bulletins/2014-june04-illicit-fentanyl-use.pdf

BC Gov News. (2016). Health. Provincial health officer declares public health emergency. Victoria, BC, 2016. Retrieved from: https://news. gov.bc.ca/releases/2016HLTH0026-000568

Buehler, J. W., Hopkins, R. S., Overhage, J. M., Sosin, D. M., \& Tong, V. (2004). Framework for evaluating public health surveillance systems for early detection of outbreaks: recommendations from the CDC Working Group. MMWR Recomm Reports, 53(RR05), 1-11. Retrieved from: http://www.cdc.gov/mmwr/preview/mmwrhtml/ rr5305al.htm

Buxton, J. A., Omura, J., Kuo, M., Ross, C., Tzemis, D., Purssell, R., Gardy, J., \& Carleton, B. (2015). Genetic determinants of cocaine-associated agranulocytosis. BMC Res Notes, 8, 240. Retrieved from: http:// www.ncbi.nlm.nih.gov/pmc/articles/PMC4467676/

Buxton, J. A., Sebastian, R., Clearsky, L., Angus, N., Shah, L., Lem, M., \& Spacey, S. D. (2011). Chasing the dragon-Characterizing cases of leukoencephalopathy associated with heroin inhalation in British Columbia. Harm Reduction Journal, 8, 3. Retrieved from: http:// www.harmreductionjournal.com/content/8/1/3

Canadian Centre on Substance Abuse. (2014. Feburary). CCENDU alert: increasing availability of counterfeit oxycodone tablets containing fentanyl. Retrieved from: http://www.ccsa.ca/Resource Library/ CCSA-CCENDU-Oxycontin-Fentanyl-Alert-2014-en.pdf

Canadian Centre on Substance Abuse. (2013, June). CCENDU drug alert illicit fentanyl. Retrieved from: http://www.ccsa.ca/Resource\%20 Library/CCSA-CCENDU-Drug-Alert-Illicit-Fentanyl-2013-en.pdf

Canadian Centre on Substance Use and Addiction. (n.d.) Canadian community epidemiology network on drug use. Retrieved from: http:// www.ccdus.ca/Eng/collaboration/CCENDU/Pages/default.aspx. 
Danaher, A. (2011). Reducing health inequities: enablers and barriers to inter-sectoral collaboration. Toronto: Wellesley Institute. Retrieved from: http://www.wellesleyinstitute.com/wp-content/ uploads/2012/09/Reducing-Health-Inequities-Enablers-and-Barriersto-Intersectoral-Collaboration.pdf

Eibl, J. K., Morin, K., Leinonen, E., \& Marsh, D. C. (2017). The state of opioid agonist therapy in Canada 20 years after federal oversight. The Canadian Journal of Psychiatry, 62(7), 444-450.

German RR. (2001). Updated guidelines for evaluating public health surveillance systems. MMWR Recomm Reports, 50(RR13), 1-35. Retrieved from: http://www.cdc.gov/mmwr/preview/mmwrhtml/ rr5013al.htm

Green, T. C., Gilbert, M. (2016). Counterfeit medications and fentanyl. JAMA Internal Medicine, 176(10), 1555-1557.

Greer, A. M., Luchenski, S. A., Amlani, A. A., Lacroix, K., Burmeister, C., \& Buxton, J. A. (2016). Peer engagement in harm reduction strategies and services: a critical case study and evaluation framework from British Columbia, Canada. BMC Public Health, 16(1), 452.

Higashikawa, Y., \& Suzuki, S. (2008). Studies on 1-(2-phenethyl)-4( $\mathrm{N}$-propionylanilino) piperidine (fentanyl) and its related compounds. VI. Structure-analgesic activity relationship for fentanyl, methyl-substituted fentanyls and other analogues. Forensic Toxicology, 26, 1-5.

Irvine, M. A., Buxton, J. A., Otterstatter, M., Balshaw, R., Gustafson, R., Tyndall, M., Kendall, P., Kerr, T., Gilbert, M., \& Coombs, D. (2018). Impact of take-home opioid agonist kit distribution during a synthetic opioid epidemic in British Columbia, Canada; a modelling study. Lancet Public Health 3(5), e218-e225. Retrieved from: http://www.thelancet.com/ pdfs/journals/lanpub/PIIS2468-2667/18)30044-6.pdf

Klar, S. A., Brodkin, E., Gibson, E., Padhi, S., Predy, C., Green, C., \& Lee, V. (2016). Notes from the field: furanyl-fentanyl overdose events caused by smoking contaminated crack cocaine - British Columbia, Canada, July 15-18. MMWR 65(37), 1015-1016. Retrieved from: https://www.cdc.gov/mmwr/volumes/65/wr/mm6537a6.htm

Know your source? Be drug smart. Retrieved from: https://knowyoursource.ca/
Knowles, L., Buxton, J. A., Skuridina, N., Achebe, I., LeGatt, D., Fan, S. Zhu, N., \& Talbot, J. (2009). Levamisole-tainted cocaine causing severe neutropenia in Alberta and British Columbia. Harm Reduction Journal, 6(1), 30. Retrieved from: http://www.harmreductionjournal. $\mathrm{com} /$ content/6/1/30

Marshall, B. D., Milloy, M. J., Wood, E., Montaner, J. S., \& Kerr, T. (2011). Reduction in overdose mortality after the opening of North America's first medically supervised safer injecting facility: a retrospective population-based study. Lancet, 377(9775), 1429-1437. doi:10.1016/50140-6736(10)62353-7

Ministry of Public Safety and Solicitor General. (2018, December 27). $\mathrm{BC}$ Coroners service. Illicit drug overdose deaths in BC January 1 , 2008-November 30, 2018. Retrieved from: https://www2.gov. bc.ca/assets/gov/birth-adoption-death-marriage-and-divorce/ deaths/coroners-service/statistical/illicit-drug.pdf

Soukup-Baljak, Y., Greer, A. M., Amlani, A., Sampson, O., \& Buxton, J. A. (2015). Drug quality assessment practices and communication of drug alerts among people who use drugs. International Journal of Drug Policy 26(12), 1251-1257.

Toward the Heart. (n.d.) Naloxone Programs. Naloxone saves lives. Retrieved from: https://towardtheheart.com/naloxone

Vancouver Police Department. (2014a, October 12). Police warn about rash of heroin overdoses. Retrieved from: http://mediareleases. vpd.ca/2014/10/12/police-warn-about-rash-of-heroin-overdoses/

Vancouver Police Department. (2014b, October 15). Update: Police warn about rash of overdoses. Retrieved from: https://mediareleases.vpd. ca/2014/10/15/update-police-warn-about-rash-of-overdoses/

Wallace, B., Pagan, F., \& Pauly, B. B. (2019). The implementation of overdose prevention sites as a novel and nimble response during an illegal drug overdose public health emergency. International Journal of Drug Policy, 66, 64-72.

Young, M. M., Pirie, T., Buxton, J. A., \& Hosein, F. S. (2015). The rise of overdose deaths involving fentanyl and the value of early warning. Canadian Journal of Addiction 6(3), 13-17. 